\title{
Resolution of Some Open Problems Concerning Multiple Zeta Evaluations of Arbitrary Depth
}

\author{
DOUGLAS BOWMAN ${ }^{1, \star}$ and DAVID M. BRADLEY ${ }^{2, \star \star}$ \\ ${ }^{1}$ Department of Mathematics, Department of Mathematical Sciences, Northern Illinois \\ University,Dekalb, IL 60115,U.S.A.e-mail:bowman@math.niu.edu \\ ${ }^{2}$ Department of Mathematics and Statistics, University of Maine, 5752 Neville Hall, Orono, ME \\ 04469-5752,U.S.A. e-mail: bradley@math.umaine.edu
}

(Received: 20 November 2001; accepted 21 February 2002)

\begin{abstract}
We prove some new evaluations for multiple polylogarithms of arbitrary depth. The simplest of our results is a multiple zeta evaluation one order of complexity beyond the wellknown Broadhurst-Zagier formula. Other results we provide settle three of the remaining outstanding conjectures of Borwein, Bradley, and Broadhurst. A complete treatment of a certain arbitrary depth class of periodic alternating unit Euler sums is also given.
\end{abstract}

Mathematics Subject Classifications (2000). Primary: 33B30, 34B30; Secondary: 11M99.

Key words. multiple zeta values, MZV, multiple polylogarithms, Euler sums, hypergeometric functions.

\section{Introduction}

The study of special values of multiple zeta functions concerns itself with relations between values at integer vectors $\left(s_{1}, \ldots, s_{k}\right)$ of sums of the form

$$
\zeta\left(s_{1}, \ldots, s_{k}\right):=\sum_{n_{1}>\cdots>n_{k}>0} \prod_{j=1}^{k} n_{j}^{-s_{j}},
$$

commonly referred to as multiple zeta values $[5,21,24,26]$. We are primarily interested in positive integer values of the arguments $s_{1}, \ldots, s_{k}$, in which case it is easily seen that $s_{1}>1$ is both necessary and sufficient for the sum (1) to converge.

A good deal of work on multiple zeta values has focused on the problem of determining when 'complicated' sums can be expressed in terms of 'simpler' sums. A crude but convenient measure of the complexity of the sum (1) is the number $k$ of nested summations. This is also equal to the number of arguments in the definition (1), and is called the depth. Thus, researchers are interested in determining which sums can be expressed in terms of other sums of lesser depth.

For each positive integer $k$, let $Z_{k}$ denote the set of all multiple zeta values (with positive integer arguments) of depth less than $k$. If we restrict our attention to

${ }^{\star}$ Research partially supported by NSF grant DMS-9705782.

$\star \star$ Research supported by the University of Maine summer faculty research fund. 
rational polynomial relationships, the aforementioned problem amounts to determining for each $k$ the values of the arguments $s_{1}, \ldots, s_{k}$ for which the sum (1) lies in the polynomial ring $\mathbf{Q}\left[Z_{k}\right]$. Settling this question in complete generality is currently well beyond the reach of number theory. For example, there is as yet no proof that $\zeta(5) \notin \mathbf{Q}\left[Z_{1}\right]=\mathbf{Q}$, although it is strongly suspected that $\zeta(5)$ is indeed irrational. Nevertheless, considerable progress has been made with regard to proving specific classes of reductions, even at arbitrary depth. A brief historical overview will serve to put the problem in perspective.

Apart from Euler's celebrated depth-1 evaluation for the Riemann zeta function

$$
\zeta(2 n)=\sum_{j=1}^{\infty} \frac{1}{j^{2 n}}=-\frac{1}{2} \frac{(2 \pi i)^{2 n} B_{2 n}}{(2 n) !}, \quad 0 \leqslant n \in \mathbf{Z},
$$

in terms of the Bernoulli numbers $B_{0}=1, B_{1}=-1 / 2, B_{2}=1 / 6, B_{3}=0, B_{4}=-1 / 30$, etc., defined by

$$
\frac{z}{\mathrm{e}^{z}-1}=\sum_{n=0}^{\infty} \frac{B_{n}}{n !} z^{n}, \quad|z|<2 \pi,
$$

the study of multiple zeta values can be fairly said to have begun with Euler's depth-2 reduction [15]

$$
2 \zeta(n, 1)=n \zeta(n+1)-\sum_{k=1}^{n-2} \zeta(n-k) \zeta(k+1), \quad 2 \leqslant n \in \mathbf{Z},
$$

expressing an infinite class of multiple zeta values of depth 2 in terms of depth-1 (Riemann zeta) values. More generally, we refer to a relation amongst multiple zeta values as a depth-k reduction if the relation expresses a multiple zeta value of depth $k$ in terms of multiple zeta values of depth less than $k$.

The first systematic study of reductions up to depth 3 was carried out by Borwein, Bailey and Girgensohn, in a short series of papers [2, 3, 7] appearing in the early 1990s. Research undertaken by Hoffman [18-21], Zagier [26], and Borwein-BradleyBroadhurst [4] on multiple zeta values of arbitrary depth led to the discovery of several relations satisfied by them. These relations can be exploited by computer algebra systems to prove reductions of small weight [23]. (Here the weight of the multiple zeta value (1) is simply the sum of the arguments: $s_{1}+s_{2}+\cdots+s_{k}$.)

Additionally, high-precision evaluation of specific multiple zeta values combined with human-directed computer searches using lattice basis reduction algorithms led to several beautiful conjectures concerning arbitrary depth reductions [4-6]. An example of an arbitrary depth reduction is

$$
\zeta(\underbrace{3,1,3,1, \ldots, 3,1}_{2 n})=4^{-n} \zeta(\underbrace{4,4, \ldots, 4}_{n})=\frac{2^{1-4 n} \zeta(4 n)}{(2 n+1)(4 n+1)\left|B_{4 n}\right|}=\frac{2 \pi^{4 n}}{(4 n+2) !},
$$

in which the positive integers $2 n$ and $n$ beneath the underbraces in (3) denote the depth of the respective multiple zeta values. The formula (3), originally conjectured 
by Zagier [26], was first proved by Broadhurst. A modification of Broadhurst's proof appears in [5]. Subsequently, purely combinatorial proofs were given $[6,10]$ based on the well-known shuffle property of iterated integrals. To simplify the reading of such formulas, when a string of arguments is repeated an exponent is used. In other words, we treat string multiplication as concatenation. With this notation, the first two members of formula (3) may be written $\zeta\left(\{3,1\}^{n}\right)=4^{-n} \zeta\left(\{4\}^{n}\right)$.

Although we focus here on analytic aspects of ultimately periodic multiple polylogarithms, other aspects also play an important role in related work. See $[6,8,10,11,13,14,16,17]$ for connections with quantum groups, motivic Lie algebras, algebraic geometry, knot theory, quantum field theory, and shuffle combinatorics, respectively. The survey [9] provides additional references and pointers to the literature.

\section{Statement of Results}

The Broadhurst-Zagier formula (3) is an example of an arbitrary depth reduction in which the argument strings are periodic. Whereas the case of periodic strings of period 1 is quite well understood - there is a formula expressing $\zeta\left(\{s\}^{n}\right)$ in terms of the depth-1 zeta values $\zeta(s), \zeta(2 s), \ldots, \zeta(n s)$ [4] very little is known in general about strings with longer periods apart from the fact that their associated bivariate generating functions satisfy a differential equation of order equal to the weight of the period. In this paper, we offer some new multiple zeta evaluations for ultimately periodic strings of period 2 and period weight 4 . We also give a complete treatment of a certain class of ultimately periodic alternating unit Euler sums of period 2. These results are highlighted in subsection 2.2 below. Three of our results settle conjectures from [4] and [5].

\subsection{ADDITIONAL NOTATION}

Let $x$ be a real number satisfying $0 \leqslant x<1$. The parametrized multiple zeta function

$$
\zeta_{x}\left(s_{1}, \ldots, s_{k}\right):=\sum_{n_{1}>\cdots>n_{k}>0} x^{n_{1}} \prod_{j=1}^{k} n_{j}^{-s_{j}}
$$

is defined for positive integers $s_{j}$, and is an instance of a multiple polylogarithm $[5,9,10]$. Of course, if $s_{1}>1$, then we can allow $x=1$ and in that case, (4) coincides with (1). Euler sums have the form

$$
\zeta\left(s_{1}, \ldots, s_{k}\right):=\sum_{n_{1}>\cdots>n_{k}>0} \prod_{j=1}^{k} n_{j}^{-\left|s_{j}\right|} \sigma_{j}^{-n_{j}},
$$

where $s_{1}, \ldots, s_{k}$ are non-zero integers and $\sigma_{j}:=\operatorname{signum}\left(s_{j}\right)$. Thus, a multiple zeta value is an Euler sum with no alternations, i.e. each $\sigma_{j}=1$. If each $s_{j}= \pm 1$, we also refer to (5) as a unit Euler sum. To avoid confusion with the notion of analytic 
continuation, we shall henceforth adopt the notation of [4], in which each $s_{j}$ in (5) is replaced by $\overline{-s_{j}}$ when $s_{j}<0$. Thus, for example, $\zeta(\overline{1})=-\log 2$.

Let $a, b, c$ and $x$ be complex numbers with $|x|<1$ and $c$ not equal to zero or a negative integer. We denote the Gaussian hypergeometric function by

$$
F(a, b ; c ; x)={ }_{2} F_{1}\left(\begin{array}{c}
a, b \\
c
\end{array} \mid x\right)=\sum_{n=0}^{\infty} \frac{(a)_{n}(b)_{n}}{(c)_{n}} \frac{x^{n}}{n !}
$$

where

$$
(a)_{n}:= \begin{cases}\prod_{j=0}^{n-1}(a+j), & \text { if } n \text { is a positive integer, } \\ 1, & \text { if } n=0\end{cases}
$$

is the Pochammer symbol (rising factorial). If $\Re(c-b-a)>0$, the series (6) converges even when $|x|=1$, and thus for complex $z$ we may abbreviate

$$
\begin{aligned}
& Y_{1}(x, z):=F(z,-z ; 1 ; x), \quad|x| \leqslant 1, \\
& Y_{2}(x, z):=(1-x) F(1+z, 1-z ; 2 ; 1-x), \quad|1-x|<1 .
\end{aligned}
$$

Also, for complex $z$ not a positive integer multiple of \pm 1 or $\pm i$, we set

$$
G(z):=\frac{1}{4}\{\psi(1+i z)+\psi(1-i z)-\psi(1+z)-\psi(1-z)\},
$$

where $\psi=\Gamma^{\prime} / \Gamma$ is the logarithmic derivative of the Euler gamma function. Finally, for any nonnegative integer $k$, power series coefficient extraction will be performed throughout by $\left[t^{k}\right] \sum_{n=0}^{\infty} a_{n} t^{n}=a_{k}$. The notation will not be confused with the truncated square brackets in $\lfloor x\rfloor$, which we use to denote the greatest integer not exceeding the real number $x$.

\subsection{MAIN RESULTS}

Our signal result is the following identity for the generating function of the ultimately periodic sequence of multiple polylogarithms $\left\{\zeta_{x}\left(3,\{1,3\}^{n}\right): 0 \leqslant n \in \mathbf{Z}\right\}$.

PROPOSITION 1 . For each real $x$ satisfying $0 \leqslant x \leqslant 1$, the formal power series

$$
S(x, z):=\sum_{n=0}^{\infty}(-1)^{n} z^{4 n+2} 4^{n} \zeta_{x}\left(3,\{1,3\}^{n}\right)
$$

defines an entire function of the complex variable z. Furthermore, if $Y_{1}, Y_{2}$ and $G$ are defined as in (7), (8) and (9), then we have the identity

$$
S(x, z)=G(z) Y_{1}(x, z) Y_{1}(x, i z)-\frac{Y_{1}(x, i z) Y_{2}(x, z)}{4 Y_{1}(1, z)}+\frac{Y_{1}(x, z) Y_{2}(x, i z)}{4 Y_{1}(1, i z)}
$$

for all pairs $(x, z)$ for which the right-hand side is defined. 
Proposition 1 has several interesting consequences, two of which are given by Theorems 1 and 2 below. Our first theorem gives a multiple zeta value reduction which seems to have escaped the extensive numerical and symbolic searches carried out by [4] and [5].

THEOREM 1. For all nonnegative integers $n$,

$$
\begin{aligned}
\zeta\left(3,\{1,3\}^{n}\right) & =4^{-n} \sum_{k=0}^{n}(-1)^{k} \zeta(4 k+3) \zeta\left(\{4\}^{n-k}\right) \\
& =\sum_{k=0}^{n} \frac{2 \pi^{4 k}}{(4 k+2) !}\left(-\frac{1}{4}\right)^{n-k} \zeta(4 n-4 k+3) .
\end{aligned}
$$

THEOREM 2 (Conjectured in [4] and [5]). For all nonnegative integers $n$,

$$
\begin{aligned}
& \zeta\left(2,\{1,3\}^{n}\right) \\
& \quad=4^{-n} \sum_{k=0}^{n}(-1)^{k} \zeta\left(\{4\}^{n-k}\right)\left\{(4 k+1) \zeta(4 k+2)-4 \sum_{j=1}^{k} \zeta(4 j-1) \zeta(4 k-4 j+3)\right\} .
\end{aligned}
$$

We have also obtained cognate results for alternating unit Euler sums $[4,5]$ and multiple polylogarithms $[5,9,16]$ at one-half. These results are proved in Section 4 , in which we settle two conjectures from [4] and as a consequence obtain reductions for the arbitrary depth alternating unit Euler sums $\zeta\left(\{\overline{1}, 1\}^{n}\right), \zeta\left(\overline{1},\{1, \overline{1}\}^{n}\right)$, $\zeta\left(\overline{1},\{\overline{1}, 1\}^{n}\right)$, and $\zeta\left(\overline{1}, \overline{1},\{1, \overline{1}\}^{n}\right)$, where $n$ is any nonnegative integer. The main result from which these reductions are derived is Proposition 2 below.

Recall the generating function

$$
A(z):=\sum_{n=0}^{\infty} z^{n} \zeta\left(\{\overline{1}\}^{n}\right)=\prod_{j=1}^{\infty}\left(1+\frac{(-1)^{j} z}{j}\right)=\frac{\Gamma(1 / 2)}{\Gamma(1+z / 2) \Gamma(1 / 2-z / 2)}
$$

from [4].

PROPOSITION 2. For each real $x$ satisfying $0 \leqslant x \leqslant 1$, the formal power series

$$
M(x, t):=\sum_{n=0}^{\infty}\left[t^{2 n} \zeta_{x}\left(\{\overline{1}, 1\}^{n}\right)+t^{2 n+1} \zeta_{x}\left(\overline{1},\{1, \overline{1}\}^{n}\right)\right]
$$

defines an entire function of the complex variable t. Furthermore, if we put $z=$ $(1+i) t / 2, s=(1+x) / 2$, and let $U(s, z)=Y_{1}(s, z)-z Y_{2}(s, z)$, where $Y_{1}$ and $Y_{2}$ are given by (7) and (8) respectively, then

$$
M(x, t)=\frac{U(s,-z) U(s, i z)}{A(-z) A(i z)}
$$

for all pairs $(x, t)$ for which the right-hand side is defined. 
From Proposition 2 we obtain the following generating function identities for alternating unit Euler sums.

COROLLARY 1 (Conjectured in [4]). Let A(z) be as in (12). Then for all complex numbers $t$, we have

$$
\sum_{n=0}^{\infty}\left[t^{2 n} \zeta\left(\{\overline{1}, 1\}^{n}\right)+t^{2 n+1} \zeta\left(\overline{1},\{1, \overline{1}\}^{n}\right)\right]=A\left(\frac{t}{1-i}\right) A\left(\frac{t}{1+i}\right)
$$

COROLLARY 2 [Conjectured in [4]). Let the functions $G$ and $A$ be given by (9) and (12), respectively. Then

$$
\begin{aligned}
1+ & \sum_{n=0}^{\infty}\left[t^{2 n+1} \zeta\left(\overline{1},\{\overline{1}, 1\}^{n}\right)+t^{2 n+2} \zeta\left(\overline{1}, \overline{1},\{1, \overline{1}\}^{n}\right)\right] \\
& =\frac{1}{2}(1+i) z A(z) A(-i z)\{\pi \csc (\pi z)-i \pi \operatorname{csch}(\pi z)+4 G(z)\},
\end{aligned}
$$

holds for all complex numbers $t$ and $z$ satisfying $z=(1+i) t / 2$ and such that the righthand side is defined.

Remark. It is routine to check that the right hand side of (15) and the corresponding expression in equation (16) of [4] are equal. Our expression has the advantage of being easier to express as a Maclaurin series.

From Corollaries 1 and 2 we obtain the following reductions for alternating unit Euler sums:

THEOREM 3. Define a sequence of numbers $c_{0}, c_{1}, \ldots \in \mathbf{Q}[\log 2, \zeta(2), \zeta(3), \ldots]$ by

$$
\sum_{m=0}^{\infty} c_{m} x^{m}=\exp \left(\sum_{k=1}^{\infty} b_{k} \frac{x^{k}}{k}\right)
$$

where $b_{1}=-\log 2$ and

$$
b_{k}=\left\{\begin{array}{l}
(-1)^{\lfloor(k+1) / 4\rfloor} 2^{(1-k) / 2}\left(2^{1-k}-1\right) \zeta(k), \quad \text { if } 1<k \text { is odd }, \\
(-1)^{1+k / 4} 2^{1-k / 2 \zeta(k),} \text { if } k \equiv 0 \bmod 4, \\
0, \quad \text { if } k \equiv 2 \bmod 4 .
\end{array}\right.
$$

Then for all nonnegative integers $n$ we have

$$
\zeta\left(\{\overline{1}, 1\}^{n}\right)=c_{2 n} \text { and } \zeta\left(\overline{1},\{1, \overline{1}\}^{n}\right)=c_{2 n+1} .
$$

THEOREM 4. Let $b_{k}$ be as in Theorem 3. Define numbers $c_{m}^{\prime} \in \mathbf{Q}[\log 2, \zeta(2)$, $\zeta(3), \ldots]$ by

$$
\sum_{m=0}^{\infty} c_{m}^{\prime} x^{m}=\left(\sum_{k=0}^{\infty} d_{k} x^{k}\right) \exp \left(\sum_{k=1}^{\infty} b_{k} \frac{x^{k}}{k}\right)
$$


where $d_{0}=1$ and

$$
d_{k}=\left\{\begin{array}{l}
(-1)^{\lfloor k+1 / 2\rfloor} 2^{2-3 k / 2}\left(2^{k-1}-1\right) \zeta(k), \quad \text { if } 0<k \text { is even }, \\
0, \quad \text { if } k \equiv 1 \bmod 4, \\
(-1)^{(k+1) / 4} 2^{(3-k) / 2} \zeta(k), \quad \text { if } k \equiv 3 \bmod 4 .
\end{array}\right.
$$

Then for all nonnegative integers $n$ we have

$$
\zeta\left(\overline{1},\{\overline{1}, 1\}^{n}\right)=c_{2 n+1}^{\prime} \quad \text { and } \zeta\left(\overline{1}, \overline{1},\{1, \overline{1}\}^{n}\right)=c_{2 n+2}^{\prime} .
$$

Remark. Of course, one can express the numbers $c_{m}$ and $c_{m}^{\prime}$ of Theorems 3 and 4 explicitly. Thus,

$$
c_{m}^{\prime}=\sum_{k=0}^{m} c_{k} d_{m-k} \quad \text { and } \quad c_{m}=\sum \prod_{k \geqslant 1} \frac{1}{j_{k} !}\left(\frac{b_{k}}{k}\right)^{j_{k}}
$$

where the sum is over all nonnegative integers $j_{1}, j_{2}, \ldots$ satisfying $\sum_{k \geqslant 1} k j_{k}=m$.

\section{Multiple Zeta Values of Period 2}

This section contains the proofs of our results pertaining to ultimately periodic multiple zeta values of period 2 and period weight 4, namely Proposition 1 and Theorems 1 and 2. It is interesting to note that the proof of Propositions 1 and 2 relies on a general result which also eliminates the need for computer algebra in the orginal proof of the Broadhurst-Zagier formula in [5] (see Lemma 1 below).

\subsection{PROOF OF PROPOSITION 1}

Let $R(x, z)$ denote the right-hand side of (11). For each fixed $x$ satisfying $0<x \leqslant 1$, $R(x, z)$ is evidently an analytic function of $z$, apart from isolated singularities (possible poles) at the positive integer multiples of $\pm 1, \pm i$. Let $n$ be a positive integer and let $p \in\{1,-1, i,-i\}$. A straightforward calculation shows that $\lim _{z \rightarrow p n}(z-p n) R(x, z)=0$ follows from the identity

$$
Y_{1}(x, n)+n(-1)^{n} Y_{2}(x, n)=0, \quad 1 \leqslant n \in \mathbf{Z},
$$

which in turn is a consequence of the identity [25, p. 254]

$$
\begin{aligned}
& \frac{(1+\alpha)_{n}}{n !}{ }_{2} F_{1}\left(\begin{array}{c|c}
-n, 1+\alpha+\beta+n \\
1+\alpha
\end{array} \mid \frac{1-y}{2}\right) \\
& =\frac{(-1)^{n}(1+\beta)_{n}}{n !}{ }_{2} F_{1}\left(\begin{array}{c|c}
-n, 1+\alpha+\beta+n \\
1+\beta
\end{array} \mid \frac{1+y}{2}\right)
\end{aligned}
$$

for the Jacobi polynomials. Thus, for $0<x \leqslant 1$, the singularities of $R(x, z)$ are all removable.

It now suffices to show that $S(x, z)$ and $R(x, z)$ both have Maclaurin series in $x$ which begin

$$
z^{2} x+\frac{1}{8} z^{2} x^{2}+\frac{1}{27} z^{2}\left(1-2 z^{4}\right) x^{3}+\frac{1}{64} z^{2}\left(1-\frac{7}{2} z^{4}\right) x^{4}+\mathrm{O}\left(x^{5}\right), \quad x \rightarrow 0,
$$


and are both annihilated by the differential operator

$$
D_{1}^{2} D_{0}^{2}+4 z^{4}, \quad D_{0}:=x \frac{\mathrm{d}}{\mathrm{d} x}, \quad D_{1}:=(1-x) \frac{\mathrm{d}}{\mathrm{d} x} .
$$

Checking these facts for $S(x, z)$ is a trivial exercise. Although in general $R(x, z)$ is undefined when $x=0$ because $Y_{2}(0, z)=F(1+z, 1-z ; 2 ; 1)$ diverges unless it terminates, a short calculation employing the special case

$$
\begin{aligned}
F(1 & +z, 1-z ; 2 ; x) \\
= & \frac{\Gamma(2)}{\Gamma(1+z) \Gamma(1-z)} \sum_{n=0}^{\infty} \frac{(1+z)_{n}(1-z)_{n}}{(n !)^{2}} \times \\
& \times\{2 \psi(n+1)-\psi(n+1-z)-\psi(n+1+z)-\log (1-x)\}(1-x)^{n}, \quad 0 \leqslant x<1
\end{aligned}
$$

of the formula 15.3 .10 of [1, p. 559] verifies that $R(x, z)$ possesses a Maclaurin series at $x=0$ that begins as stated.

The fact that $\left(D_{1}^{2} D_{0}^{2}+4 z^{4}\right) R(x, z)=0$ is most easily seen by setting $f(x)=1-x$, $g(x)=x$, and $t=z^{2}$ in Lemma 1 below. Two solutions to the differential equation $\left(D_{1} D_{0}+z^{2}\right) y=0$ are given by $y=Y_{1}(x, z)$ and $y=Y_{2}(x, z)$, and thus changing $z$ to $i z$ we see that two solutions of the differential equation $\left(D_{1} D_{0}-z^{2}\right) y=0$ are $y=Y_{1}(x, i z)$ and $y=Y_{2}(x, i z)$. The lemma then shows that each of the functions $Y_{1}(x, z) Y_{1}(x, i z), Y_{1}(x, i z) Y_{2}(x, z)$, and $Y_{1}(x, z) Y_{2}(x, i z)$ are annihilated by the operator (17).

LEMMA 1. Let $K$ be a field of characteristic not equal to 2 and let $D$ be a derivation on $K$. For each $k \in K$, define a derivation $D_{k}:=k D$. Let $t$ be a constant, and suppose that for some $f, g, u, v \in K$ the differential equations $\left(D_{f} D_{g}+t\right) u=0$ and $\left(D_{f} D_{g}-t\right) v=0$ hold. Then $u v$ is annhilated by the differential operator $\left(D_{f}^{2} D_{g}^{2}+4 t^{2}\right)$.

Proof. First note that $u D_{g}^{2} v+v D_{g}^{2} u=0$, for

$$
\left(u D_{g}^{2} v+v D_{g}^{2} u\right) f=\left(u D_{f} D_{g} v+v D_{f} D_{g} u\right) g=(u t v-v t u) g=0 .
$$

We now calculate $D_{f}^{2} D_{g}^{2}(u v)$. By the Leibniz rule and our note above,

$$
D_{f}^{2} D_{g}^{2}(u v)=D_{f}^{2}\left(u D_{g}^{2} v+2\left(D_{g} u\right)\left(D_{g} v\right)+v D_{g}^{2} u\right)=2 D_{f}^{2}\left(D_{g} u\right)\left(D_{g} v\right)
$$

But

$$
\begin{aligned}
D_{f}^{2}\left(D_{g} u\right)\left(D_{g} v\right) & =D_{f}\left(D_{g} u\right)\left(D_{f} D_{g} v\right)+D_{f}\left(D_{f} D_{g} u\right)\left(D_{g} v\right) \\
& =D_{f}\left(D_{g} u\right)(t v)+D_{f}(-t u)\left(D_{g} v\right) \\
& =t\left[v D_{f} D_{g} u+\left(D_{f} v\right)\left(D_{g} u\right)-\left(D_{f} u\right)\left(D_{g} v\right)-u D_{f} D_{g} v\right] .
\end{aligned}
$$

In the previous expression, the middle two terms cancel since

$$
\left(D_{f} v\right)\left(D_{g} u\right)=(f g)(D v)(D u)=\left(D_{f} u\right)\left(D_{g} v\right) .
$$


Hence, we have

$$
D_{f}^{2} D_{g}^{2}(u v)=2 t\left[v D_{f} D_{g} u-u D_{f} D_{g} v\right]=2 t[v(-t u)-u(t v)]=-4 t^{2} u v .
$$

\subsection{PROOF OF THEOREM 1}

Let $x=1$ in the identity (11) of Proposition 1 and then extract the coefficient of the appropriate power of $z$. More explicitly, we note that

$$
\zeta\left(3,\{1,3\}^{n}\right)=(-1)^{n} 4^{-n}\left[z^{4 n+2}\right] S(1, z) .
$$

But

$$
S(1, z)=G(z) Y_{1}(1, z) Y_{1}(1, i z)=G(z) Q(z),
$$

where by Gauss's ${ }_{2} F_{1}$ summation theorem and the infinite product formula for sine,

$$
Q(z):=Y_{1}(1, z) Y_{1}(1, i z)=\frac{\sin (\pi z)}{\pi z} \frac{\sin (\pi i z)}{\pi i z}=\sum_{n=0}^{\infty}(-1)^{n} z^{4 n} \zeta\left(\{4\}^{n}\right)
$$

Since

$$
G(z)=\sum_{n=0}^{\infty} z^{4 n+2} \zeta(4 n+3), \quad|z|<1,
$$

the desired formula follows from (18), (3), and the formula for the coefficient in the Cauchy product of power series.

COROLLARY 3. For all non-negative integers $n$,

$$
\begin{aligned}
\zeta\left(2,1,\{3,1\}^{n}\right) & =4^{-n} \sum_{k=0}^{n}(-1)^{k} \zeta(4 k+3) \zeta\left(\{4\}^{n-k}\right) \\
& =\sum_{k=0}^{n} \frac{2 \pi^{4 k}}{(4 k+2) !}\left(-\frac{1}{4}\right)^{n-k} \zeta(4 n-4 k+3) .
\end{aligned}
$$

Proof. Apply duality [5, 22, 26] to Theorem 1.

\subsection{PROOF OF THEOREM 2}

Differentiate both sides of (11) in Proposition 1 with respect to $x$, let $x \rightarrow 1-$ (requires asymptotic formulas for the relevant hypergeometrics) and then extract the coefficient of the appropriate power of $z$. More explicitly, we note that

$$
x \frac{\mathrm{d}}{\mathrm{d} x} S(x, z)=\sum_{n=0}^{\infty}(-1)^{n} z^{4 n+2} 4^{n} \zeta_{x}\left(2,\{1,3\}^{n}\right),
$$

and hence (letting prime denote differentiation with respect to the first argument)

$$
\zeta\left(2,\{1,3\}^{n}\right)=(-1)^{n} 4^{-n}\left[z^{4 n+2}\right] S^{\prime}(1, z) .
$$


Differentiating (11) we get that

$$
\begin{aligned}
S^{\prime}(x, z)= & G(z)\{H(x, z)+H(x, i z)\}-\frac{Y_{1}^{\prime}(x, i z) Y_{2}(x, z)}{4 Y_{1}(1, z)}- \\
& -\frac{Y_{1}(x, i z) Y_{2}^{\prime}(x, z)}{4 Y_{1}(1, z)}+\frac{Y_{1}^{\prime}(x, z) Y_{2}(x, i z)}{4 Y_{1}(1, i z)}+\frac{Y_{1}(x, z) Y_{2}^{\prime}(x, i z)}{4 Y_{1}(1, i z)},
\end{aligned}
$$

where

$$
H(x, z):=-z^{2} F(1+z, 1-z ; 2 ; x) F(i z,-i z ; 1 ; x) .
$$

Entries [15.3.10] and [15.3.11] of [1] provide the asymptotic formulas

$$
\begin{aligned}
F(1+z, 1-z ; 2 ; x)= & \frac{2 \psi(1)-\psi(1+z)-\psi(1-z)-\log (1-x)}{\Gamma(1+z) \Gamma(1-z)}+ \\
& +\mathrm{O}((1-x) \log (1-x)), \quad x \rightarrow 1-,
\end{aligned}
$$

and

$$
F(i z,-i z ; 1 ; x)=\frac{1}{\Gamma(1+i z) \Gamma(1-i z)}+\mathrm{O}((1-x) \log (1-x)), \quad x \rightarrow 1-,
$$

respectively. If we now substitute the asymptotic formulas (24) and (25) into (23), apply the reflection formula for the gamma function and the definition (19), there comes

$$
\begin{aligned}
H(x, z) & =-z^{2} \frac{\sin (\pi z)}{\pi z} \cdot \frac{\sin (\pi i z)}{\pi i z}\{2 \psi(1)-\psi(1+z)-\psi(1-z)-\log (1-x)+o(1)\} \\
& =-z^{2} Q(z)\{2 \psi(1)-\psi(1+z)-\psi(1-z)-\log (1-x)+o(1)\}
\end{aligned}
$$

and hence as $x \rightarrow 1-$,

$$
\begin{aligned}
& H(x, z)+H(x, i z) \\
&= Q(z)\left\{z^{2}(\log (1-x)-2 \psi(1)+\psi(1+z)+\psi(1-z))-\right. \\
&\left.-z^{2}(\log (1-x)-2 \psi(1)-\psi(1+i z)-\psi(1-i z))\right\}+\mathrm{o}(1) \\
&=-4 z^{2} Q(z) G(z)+\mathrm{o}(1) .
\end{aligned}
$$

We now substitute (26) into (22). Since $Y_{2}(x, z)=\mathrm{O}(1-x)$ and $Y_{1}^{\prime}(x, z)=$ $\mathrm{O}(\log (1-x))$ as $x \rightarrow 1-$, it follows that

$$
\begin{aligned}
S^{\prime}(x, z)= & -4 z^{2} G(z) Q(z) G(z)- \\
& -\frac{Y_{1}(x, i z)}{4 Y_{1}(1, z)}\{-F(1+z, 1-z ; 2 ; 1-x)+\mathrm{O}(1-x)\}+ \\
& +\frac{Y_{1}(x, z)}{4 Y_{1}(1, i z)}\{-F(1+i z, 1-i z ; 2 ; 1-x)+\mathrm{O}(1-x)\}+\mathrm{o}(1) .
\end{aligned}
$$


We now let $x \rightarrow 1-$ in (27), obtaining

$$
S^{\prime}(1, z)=-4 z^{2} Q(z) G^{2}(z)+\frac{1}{4} \pi^{2} z^{2} Q(z)\left\{\csc ^{2}(\pi z)-\operatorname{csch}^{2}(\pi z)\right\} .
$$

In view of (21), the proof of Theorem 2 now follows on extracting the coefficient of the appropriate power of $z$ from both sides of (28).

Remark. It is possible to continue differentiating (22) and obtain generating functions for the multiple polylogarithms $\zeta_{x}\left(1,\{1,3\}^{n}\right)$ and $\zeta_{x}\left(\{1,3\}^{n}\right)$. One can similarly differentiate $Y_{1}(x, z) Y_{1}(x, i z)$ and obtain generating functions for $\zeta_{x}\left(2,1,\{3,1\}^{n}\right), \zeta_{x}\left(1,1,\{3,1\}^{n}\right)$, and $\zeta_{x}\left(1,\{3,1\}^{n}\right)$. Setting $x=1 / 2$ in all these generating functions and dualizing then allows one to obtain reductions for the alternating unit Euler sums $\zeta\left(\{\overline{1}, 1\}^{n}\right), \zeta\left(\overline{1},\{1, \overline{1}\}^{n}\right), \zeta\left(\overline{1},\{\overline{1}, 1\}^{n}\right)$, and $\zeta\left(\overline{1}, \overline{1},\{1, \overline{1}\}^{n}\right)$ for all nonnegative integers $n$. The key step is to observe that the derivatives

$$
\left.\left(\frac{\mathrm{d}}{\mathrm{d} x}\right)^{k} F(z,-z ; 1 ; x)\right|_{x=1 / 2} \quad(0 \leqslant k \in \mathbf{Z})
$$

can be expressed, via entry [15.1.25] of [1], in terms of gamma functions, and thereby in terms of the generating function (12) for the sequence $\left\{\zeta\left(\{\overline{1}\}^{n}\right): 0 \leqslant n \in \mathbf{Z}\right\}$, each term of which is reducible [4]. This procedure is cumbersome, however, and in the next section we obtain the same results by a much more elegant method. In the process we settle two additional conjectures from [4].

\section{Alternating Unit Euler Sums of Period 2}

This section contains the proofs of our results pertaining to ultimately periodic alternating unit Euler sums of period 2, namely Proposition 2 and Theorems 3 and 4.

\subsection{PROOF OF PROPOSITION 2}

One first checks that $M(x, t)$ satisfies the differential equation

$$
\left[(1-x) \frac{\mathrm{d}}{\mathrm{d} x}\right]^{2}\left[-(1+x) \frac{\mathrm{d}}{\mathrm{d} x}\right]^{2} M(x, t)=t^{4} M(x, t) .
$$

To solve the differential Equation (29), one could apply Lemma 1 directly with $f(x)=1+x$ and $g(x)=1-x$. However, it is more convenient to make a change of variable. With $s=(1+x) / 2, z=(1+i) t / 2$ and $L(s, z):=M(x, t)$, (29) goes over into

$$
\left\{\left[(1-s) \frac{\mathrm{d}}{\mathrm{d} s}\right]^{2}\left[s \frac{\mathrm{d}}{\mathrm{d} s}\right]^{2}+4 z^{4}\right\} L(s, z)=0,
$$

which we've already encountered (17). A routine computation using entry [15.1.25] of [1] shows that 


$$
\begin{aligned}
& U(1 / 2, z)=A(z) \\
& \left.\frac{\mathrm{d}}{\mathrm{d} s} U(s, z)\right|_{s=1 / 2}=2 z A(z), \\
& \left.\frac{\mathrm{d}^{2}}{\mathrm{~d} s^{2}} U(s, z)\right|_{s=1 / 2}=-4 z(1+z) A(z), \\
& \left.\frac{\mathrm{d}^{3}}{\mathrm{~d} s^{3}} U(s, z)\right|_{s=1 / 2}=8 z(1+z)(2-z) A(z) .
\end{aligned}
$$

Using these relations, it is can be easily shown that the Wronskian determinant of the four functions

$$
U(s, z) U(s, i z), \quad U(s,-z) U(s, i z), \quad U(s, z) U(s,-i z), \quad U(s,-z) U(s,-i z)
$$

at $s=1 / 2$ is equal to

$$
-2^{13} z^{6}\left(\frac{\sin (\pi z)}{\pi z}\right)^{2}\left(\frac{\sinh (\pi z)}{\pi z}\right)^{2} .
$$

Since the Wronskian is not identically zero, the four functions are linearly independent. From the differential Equation (30) and Lemma 1 with $f(s)=1-s$ and $g(s)=s$, it follows that there exist functions $\alpha(z), \beta(z), \gamma(z), \delta(z)$ such that

$$
\begin{aligned}
M(x, t)=L(s, z)= & \alpha(z) U(s, z) U(s, i z)+\beta(z) U(s,-z) U(s, i z)+ \\
& +\gamma(z) U(s, z) U(s,-i z)+\delta(z) U(s,-z) U(s,-i z) .
\end{aligned}
$$

Now setting $x=0$ (which is the same as $s=1 / 2$ ) in (31), and performing the operations

$$
\begin{aligned}
& -\left.(1+x) \frac{\mathrm{d}}{\mathrm{d} x} M(x, t)\right|_{x=0}=-\left.s \frac{\mathrm{d}}{\mathrm{d} s} L(s, t)\right|_{s=1 / 2} \\
& {\left.\left[-(1+x) \frac{\mathrm{d}}{\mathrm{d} x}\right]^{2} M(x, t)\right|_{x=0}=\left.\left[-s \frac{\mathrm{d}}{\mathrm{d} s}\right]^{2} L(s, z)\right|_{s=1 / 2},} \\
& \left.(1-x) \frac{\mathrm{d}}{\mathrm{d} x}\left[-(1+x) \frac{\mathrm{d}}{\mathrm{d} x}\right]^{2} M(x, t)\right|_{x=0}=\left.(1-s) \frac{\mathrm{d}}{\mathrm{d} s}\left[-s \frac{\mathrm{d}}{\mathrm{d} s}\right]^{2} L(s, z)\right|_{s=1 / 2}
\end{aligned}
$$

yields the following system of equations:

$$
\left[\begin{array}{cccc}
1 & 1 & 1 & 1 \\
-i & 1 & -1 & i \\
-1 & 1 & 1 & -1 \\
i & 1 & -1 & i
\end{array}\right]\left[\begin{array}{c}
A(z) A(i z) \alpha(z) \\
A(-z) A(i z) \beta(z) \\
A(z) A(-i z) \gamma(z) \\
A(-z) A(-i z) \delta(z)
\end{array}\right]=\left[\begin{array}{l}
1 \\
1 \\
1 \\
1
\end{array}\right]
$$

Inspection via Cramer's rule gives $\alpha(z)=\gamma(z)=\delta(z)=0$ and $\beta(z)=1 /(A(-z) A(i z))$. It follows that

$$
M(x, t)=L(s, z)=\frac{U(s,-z) U(s, i z)}{A(-z) A(i z)},
$$

wherever the right hand side is defined. As in the proof of Proposition 1, we find that as a consequence of the Jacobi polynomial identity (16), the singularities (which in 
this case occur when $z$ is an even positive integer multiple of 1 or $i$ or an odd positive integer multiple of -1 or $-i)$ are all removable. Thus, $M(x, t)$ is an entire function of $t$ for each $x$ satisfying $0 \leqslant x \leqslant 1$.

4.1.1. Proof of Corollary 1. Set $x=1$ in Equation (14) of Proposition 2. In view of the fact that $Y_{2}(1, z)=0$, we have $U(1, z)=Y_{1}(1, z)=\sin (\pi z) /(\pi z)$. Thus,

$$
\begin{aligned}
M(1, t) & =\frac{U(1,-z) U(1, i z)}{A(-z) A(i z)}=\frac{Y_{1}(1, z) Y_{1}(1, i z)}{A(-z) A(i z)} \\
& =A(z) A(-i z) \cdot \frac{Y_{1}(1, z) Y_{1}(1, i z)}{A(z) A(-z) A(i z) A(-i z)}=A(z) A(-i z) .
\end{aligned}
$$

Remark. Broadhurst [12] has outlined a different proof of Corollary 1 using iterated integrals.

\subsection{PROOF OF THEOREM 3}

By Corollary 1 we need to compute the Maclaurin series for $A(t /(1-i)) A(t /(1+i))$. From Equation (12) of [4],

$$
A(z)=\exp \left(\sum_{k=1}^{\infty} \frac{(-1)^{k+1} a_{k} z^{k}}{k}\right)
$$

where

$$
a_{k}=\operatorname{Li}_{k}\left((-1)^{k}\right)=\sum_{n=1}^{\infty} \frac{(-1)^{n k}}{n^{k}}=\left\{\begin{array}{l}
-\log 2, \quad \text { if } k=1 \\
\zeta(k), \text { if } k \text { is even; } \\
\left(2^{1-k}-1\right) \zeta(k), \quad \text { if } k>1 \text { is odd. }
\end{array}\right.
$$

Hence

$$
A\left(\frac{t}{1-i}\right) A\left(\frac{t}{1+i}\right)=\exp \left(\sum_{k=1}^{\infty} \frac{\left[(-1)^{k+1}-i^{k}\right] a_{k} t^{k}}{k(1-i)^{k}}\right) .
$$

Simplifying the complex values in the sum we find that

$$
A\left(\frac{t}{1-i}\right) A\left(\frac{t}{1+i}\right)=\exp \left(\sum_{k=1}^{\infty} b_{k} \frac{t^{k}}{k}\right)
$$

where the sequence $b_{k}$ is as defined in the theorem. Expanding the exponential completes the proof.

Applying duality $[5,22,26]$ to Theorem 3 shows that we have also obtained reductions for certain multiple polylogarithmic values at $1 / 2$. Specifically, for all nonnegative integers $n$,

$$
\begin{aligned}
& \zeta_{1 / 2}\left(\{3,1\}^{n}\right)=\zeta\left(\{\overline{1}, 1\}^{2 n}\right), \quad \zeta_{1 / 2}\left(2,1,\{3,1\}^{n}\right)=\zeta\left(\overline{1},\{1, \overline{1}\}^{2 n+1}\right), \\
& \zeta_{1 / 2}\left(1,1,\{3,1\}^{n}\right)=\zeta\left(\{\overline{1}, 1\}^{2 n+1}\right), \quad \zeta_{1 / 2}\left(1,\{3,1\}^{n}\right)=-\zeta\left(\overline{1},\{1, \overline{1}\}^{2 n}\right) .
\end{aligned}
$$




\subsection{PROOF OF COROLLARY 2}

For each real $x$ satisfying $0 \leqslant x \leqslant 1$, form the formal power series

$$
T(x, t):=1+\sum_{n=0}^{\infty}\left[t^{2 n+1} \zeta_{x}\left(\overline{1},\{\overline{1}, 1\}^{n}\right)+t^{2 n+2} \zeta_{x}\left(\overline{1}, \overline{1},\{1, \overline{1}\}^{n}\right)\right] .
$$

Then it is a routine computation to show that

$$
T(x, t)=-\left(\frac{1+x}{t}\right) \frac{\mathrm{d}}{\mathrm{d} x} M(x, t),
$$

where $M(x, t)$ is as in Equation (13). Put

$$
z=(1+i) t / 2, \quad s=(1+x) / 2, \quad \text { and } \quad U(s, z)=Y_{1}(s, z)-z Y_{2}(s, z),
$$

where $Y_{1}$ and $Y_{2}$ are given by (7) and (8), respectively. From Proposition 2, it follows that

$$
T(x, t)=-\frac{s}{z}\left(\frac{1+i}{2}\right) \frac{U^{\prime}(s, i z) U(s,-z)+U(s, i z) U^{\prime}(s,-z)}{A(-z) A(i z)},
$$

wherever the right-hand side is defined, and where the prime denotes differentiation with respect to $s$. As in the proof of Proposition 2, we find that for each $x$ satisfying $0 \leqslant x \leqslant 1, T(x, t)$ defines an entire function of $t$.

We now compute the right hand side of (33) when $x=s=1$. The obstacle to overcome is the singularity of $Y_{1}^{\prime}(s, a z)$ at $s=1$. (We will be taking $a$ to be -1 or $i$ as needed.) Of course this function occurs twice in the generating function so that the singularities cancel. In particular, from the definitions of the functions it is immediate that

$$
U^{\prime}(s, a z)=a z-a^{2} z^{2} F(1+a z, 1-a z ; 2 ; s)+\mathrm{O}(1-s), \quad s \rightarrow 1-.
$$

By entry [15.3.10] of [1] it follows that

$$
\begin{aligned}
U^{\prime}(s, a z)= & a z-\frac{\left(a^{2} z^{2}\right)(2 \psi(1)-\psi(1+a z)-\psi(1-a z)-\log (1-s))}{\Gamma(1+a z) \Gamma(1-a z)}+ \\
& +\mathrm{O}((1-s) \log (1-s)), \quad s \rightarrow 1-.
\end{aligned}
$$

Similarly, from [15.3.11] of [1],

$$
U(s, a z)=\frac{1}{\Gamma(1+a z) \Gamma(1-a z)}+\mathrm{O}((1-s) \log (1-s)), \quad s \rightarrow 1-.
$$

Substituting these expressions (with $a$ taken to be -1 or $i$ as appropriate) into (33), applying the reflection formula for the gamma function, and simplifying yields

$$
\begin{aligned}
T(x, t)= & \frac{1}{2}(1+i) \operatorname{szA}(z) A(-i z)\{\pi \csc (\pi z)-i \pi \operatorname{csch}(\pi z)+4 G(z)\}+ \\
& +\mathrm{O}((1-s) \log (1-s)), \quad s \rightarrow 1-.
\end{aligned}
$$

Letting $s \rightarrow 1-$ completes the proof of the corollary. 


\subsection{PROOF OF THEOREM 4}

We now conclude the proof of Theorem 4. Changing variables from $z$ to $t$ in Corollary 2, using (20) and the well-known Maclaurin series for cosecant gives

$$
\begin{aligned}
1+ & \sum_{n=0}^{\infty}\left[t^{2 n+1} \zeta\left(\overline{1},\{\overline{1}, 1\}^{n}\right)+t^{2 n+2} \zeta\left(\overline{1}, \overline{1},\{1, \overline{1}\}^{n}\right)\right] \\
= & A\left(\frac{t}{1-i}\right) A\left(\frac{t}{1+i}\right) \times \\
& \times \sum_{n=0}^{\infty}\left[(-1)^{\lfloor(n+1) / 2\rfloor} 2^{2-3 n}\left(2^{2 n-1}-1\right) \zeta(2 n) t^{2 n}-(-4)^{-n} \zeta(4 n+3) t^{4 n+3}\right] .
\end{aligned}
$$

But this last expression is exactly $\sum_{k=0}^{\infty} c_{k} t^{k} \sum_{j=0}^{\infty} d_{j} t^{j}$. The theorem now follows by taking the Cauchy product and equating coefficients.

Duality $[5,22,26]$ applied to Theorem 4 provides evaluations for multiple polylogarithms at $1 / 2$. For all nonnegative integers $n$,

$$
\begin{aligned}
& \zeta_{1 / 2}\left(3,\{1,3\}^{n}\right)=-\zeta\left(\overline{1},\{\overline{1}, 1\}^{2 n+1}\right), \\
& \zeta_{1 / 2}\left(2,\{1,3\}^{n}\right)=-\zeta\left(\overline{1}, \overline{1},\{1, \overline{1}\}^{2 n}\right), \\
& \zeta_{1 / 2}\left(1,\{1,3\}^{n}\right)=-\zeta\left(\overline{1},\{\overline{1}, 1\}^{2 n}\right), \\
& \zeta_{1 / 2}\left(\{1,3\}^{n+1}\right)=\zeta\left(\overline{1}, \overline{1},\{1, \overline{1}\}^{2 n+1}\right) .
\end{aligned}
$$

\section{Acknowledgement}

We thank the anonymous referee for suggestions which led to improvements in the exposition.

\section{References}

1. Abramowitz, M. and Stegun, I. A.: Handbook of Mathematical Functions, Dover, New York, 1972.

2. Bailey, D. H., Borwein, J. M. and Girgensohn, R.: Experimental evaluation of Euler sums, Exposition Math. 3 (1994), 17-30.

3. Borwein, D., Borwein, J. M. and Girgensohn, R.: Explicit evaluation of Euler sums, Proc. Edinburgh Math. Soc. 38 (1995), 277-294.

4. Borwein, J. M., Bradley, D. M. and Broadhurst, D. J.: Evaluations of $k$-fold Euler/Zagier sums: A Compendium of results for arbitrary $k$, Electron J. Combin. 4(2) (1997), \#R5.

5. Borwein, J. M., Bradley, D. M., Broadhurst, D. J. and Lisoněk, P.: Special values of multiple polylogarithms, Trans. Amer. Math. Soc. 355(3) (2001), 907-941.

6. Borwein, J. M., Bradley, D. M., Broadhurst, D. J. and Lisoněk, P.: Combinatorial aspects of multiple zeta values, Electron J. Combin. 5(1) (1998), \#R38.

7. Borwein, J. M. and Girgensohn, R.: Evaluation of triple Euler sums, Electron J. Combin. 3 (1996) \#R23.

8. Bowman, D. and Bradley, D. M.: The algebra and combinatorics of shuffles and multiple zeta values, to appear in J. Combin. Theory A. 
9. Bowman, D. and Bradley, D. M.: Multiple polylogarithms: A brief survey, In: B. C. Berndt and K. Ono (eds), Proc. Conference on q-Series with Applications to Combinatorics, Number Theory and Physics, Contemp. Math. 291, Amer. Math. Soc., Providence, 2001, pp. 71-92.

10. Bowman, D., Bradley, D. M. and Ryoo, J. H.: Some multi-set inclusions associated with shuffle convolutions and multiple zeta values, submitted June 2001.

11. Broadhurst, D. J.: On the enumeration of irreducible $k$-fold Euler sums and their roles in knot theory and field theory, to appear in J. Math. Phys.

12. Broadhurst, D. J.: private e-mail, 1997.

13. Broadhurst, V. J. and Kreimer, D.: Association of multiple zeta values with positive knots via Feynman diagrams up to 9 Loops, Phys. Lett. B 393 (1997), 403-412.

14. Drinfeld, V. G.: On quasitriangular quasi-Hopf Algebras and a group closely connected with $\operatorname{Gal}(\overline{\mathbf{Q}} / \mathbf{Q})$, Algebra i Analiz, 2(4) (1990), 149-181, English transl.: Lenningrad Math. J. 2 (1991), 829-860. [MR 92f:16047]

15. Euler, L.: Meditationes circa singulare serierum genus, Novi Comm. Acad. Sci. Petropol. 20 (1775), 140-186; reprinted in Opera Omnia, ser. I, vol. 15, B. G. Teubner, Berlin, 1927, pp. 217-267.

16. Goncharov, A. B.: Multiple polylogarithms, cyclotomy and modular complexes, Math. Res. Lett. 5(4) (1998), 497-516.

17. Goncharov, A. B.: Polylogarithms in arithmetic and geometry, Proc. ICM-94, Zurich, 1995, pp. 374-387.

18. Hoffman, M. E.: Multiple harmonic series, Pacific J. Math. 152(2) (1992), 275-290.

19. Hoffman, M. E.: The algebra of multiple harmonic series, J. Algebra 194 (1997), 477-495.

20. Hoffman, M. E.: Quasi-shuffle products, J. Algebraic Combin. 11 (2000), 49-68.

21. Hoffman, M. E.: Algebraic structures on the set of multiple zeta values, Preprint.

22. Kassel, C.: Quantum Groups, Springer-Verlag, New York, 1995.

23. Minh, H. N. and Petitot, M.: Polylogarithms and the Riemann $\zeta$ function, Discrete Math. 217(1-3) (2000), 273-292.

24. Ohno, Y.: A generalization of the duality and sum formulas on the multiple zeta values, J. Number Theory 74 (1999), 39-43.

25. Rainville, E. D.: Special Functions, Chelsea Publishing, New York, 1971.

26. Zagier, D.: Values of zeta functions and their applications, In: First Europ. Congress of Mathematics, Vol. II, Birkhäuser, Boston, 1994, pp. 497-512. 